\title{
Small cell lung cancer growth is inhibited by miR-342 through its effect of the target gene IA-2
}

\author{
Huanyu Xu ${ }^{\dagger}$, Tao Cai ${ }^{\dagger}$, Gilberto N. Carmona, Liron Abuhatzira and Abner L. Notkins ${ }^{*}$
}

\begin{abstract}
Background: Small cell lung cancers (SCLC) are tumors of neuroendocrine origin. Previous in vitro studies from our laboratory showed that SCLC expresses high levels of the transmembrane dense core vesicle protein IA-2 (islet cell antigen-2) as compared to normal lung cells. IA-2, through its effect on dense core vesicles (DCVs), is known to be involved in the secretion of hormones and neurotransmitters. It is believed that the dysregulated release of the neurotransmitter Acetylcholine (ACh) by DCVs has an autocrine effect on SCLC cell growth. Recently, we found that IA- 2 is a target of the microRNA miR-342 and that miR-342 mimics suppress the expression of IA-2. The present experiments were initiated to see whether IA-2 and/or miR-342 affect the growth of SCLC.

Methods: SCLC cell growth was evaluated following the knockdown of endogenous IA-2 with RNAi or by overexpressing miR-342 with a mimic. The secretion and content of ACh in SCLC cells was analyzed using a human acetylcholine ELISA (enzyme-linked immunosorbent assay) kit.

Results: The knockdown of endogenous IA-2 by RNAi reduced SCLC cell growth within 4 days by $40 \%$ or more. Similar results were obtained when these cell lines were transfected with a miR-342 mimic. The knockdown of IA-2 by RNAi or miR-342 with a mimic also resulted in a significant decrease in the secretion of ACh, one of the autocrine hormones secreted by SCLC. Further studies revealed that the growth of SCLC cell lines that had been treated with the miR-342 mimic was restored to nearly normal levels by treatment with ACh.

Conclusion: Our studies show for the first time that both miR-342 and its target gene IA-2 are involved in the growth process of SCLC cells and act by their effect on autocrine secretion. These findings point to possible new therapeutic approaches for the treatment of autocrine-induced tumor proliferation.
\end{abstract}

Keywords: Small cell lung cancers (SCLC), Dense-core vesicle (DCV), microRNA, Autocrine, Therapeutic approach

\section{Background}

Lung cancer is divided into two histopathological types: non-small cell lung cancer (NSCLC) accounting for $\sim 80$ to $85 \%$ of lung cancers, and small cell lung cancer (SCLC) which accounts for $\sim 15$ to $20 \%$ of lung cancers [1-4]. SCLC has distinctive neuroendocrine features [5] and is capable of secreting or co-secreting a variety of neuropeptides and neurotransmitters including acetylcholine

\footnotetext{
*Correspondence: anotkins@dir.nidcr.nih.gov

†Huanyu Xu and Tao Cai contributed equally to this work

Experimental Medicine Section, Laboratory of Sensory Biology, National Institute of Dental and Craniofacial Research (NIDCR), National Institutes of Health (NIH), B30/Rm106, Bethesda, MD 20892, USA
}

(ACh) [6, 7], pro-opiomelanocortin (POMC), and adrenocorticotropic hormone (ACTH) $[8,9]$. Of the various neuropeptides, $\mathrm{ACh}$, which is an autocrine growth factor, facilitates SCLC growth $[6,7]$.

IA-2 (also known as islet cell antigen 512 [ICA512] or protein tyrosine phosphatase, receptor type $\mathrm{N}$ [PTPRN]) is an integral transmembrane protein of dense core vesicles (DCV) $[10,11]$, and plays an important role in the secretion of hormones and neurotransmitters such as insulin, luteinizing hormone ( $\mathrm{LH})$, follicular stimulating hormone (FSH), norepinephrine (NE), dopamine, and renin [10, 12-17]. In addition, IA-2 is highly expressed in tumors of neuroendocrine origin $[18,19]$, including 
SCLC tumors, as observed by northern blot analysis $[18,19]$. IA-2, together with its paralog protein IA-2 $\beta$ (also known as phosphatase homologue in granules of insulinoma [Phogrin] or protein tyrosine phosphatase, receptor type N2 [PTPRN2]), has been implicated in the growth of pancreatic $\beta$ cells $[20,21]$. Collectively, these studies suggest that IA-2 may be associated with the pathogenesis of SCLC, specifically in respect to the regulation of the neuroendocrine secretion.

The role of microRNAs (miRNAs) in tumor growth is being widely studied [22]. It is believed that the dysregulation of some miRNAs is linked to lung cancer, and that quantification of miRNA expression can be used to study disease prognosis. In this context, a recent microarray analysis showed that miR-342 is one of the most downregulated miRNAs associated with lung cancer [23]. The underlying role of miR-342 in the pathogenesis of lung cancer, however, remains unclear.

In the present study, we measured the levels of IA-2 and miR-342 in two SCLC cell lines, and then inhibited IA-2 by siRNA transfection and altered miR-342 levels with mimics and inhibitors to determine their effect on SCLC growth and ACh levels.

\section{Methods}

\section{Reagents and cell lines}

Predesigned siRNAs (Hs_PTPRN_3, 4, 5, 6) against human IA-2 (NM_001199763, NM_001199764, NM_002846), scrambled negative control, miR-342 mimic, miR-342 inhibitor, and HiPerFect Transfection Reagent were obtained from Qiagen. For RNA analysis: miRNeasy Mini kit, miScript II RT kit, and miScript SYBR Green PCR kit also were purchased from Qiagen. SYBR Green PCR Master Mix was obtained from Applied Biosystems. The human Acetylcholine (ACh) ELISA Kit was used for determination of endogenous ACh levels (MyBioSource, San Diego, CA). The Cell Counting Kit-8, neostigmine, and Acetylcholine chloride were purchased from SigmaAldrich, and the M-PER Mammalian Protein Extraction Reagent was obtained from Thermo Fisher Scientific.

Antibodies for western blotting and immunostaining were purchased from Invitrogen. VECTASHIELD Mounting Medium with DAPI (4',6-Diamidino-2-Phenylindole, Dihydrochloride) was obtained from Vector Laboratories. Rabbit polyclonal antibody against amino acid residues $65-80$ of the $\mathrm{N}$-terminal region of IA-2 (GenBank Acc. No., NP_001186692), was used for both Western blotting (1:500) and immunostaining (1:100). Both items were purchased from Abgent, San Diego, CA, USA [24].

SCLC cell lines (NCI-H82 and NCI-H345) were purchased from ATCC. The NCI-H82 cell line was cultured in Dulbecco's Modified Eagle Medium (DMEM) supplemented with $10 \%$ fetal bovine serum (FBS). The NCI-H345 cell line was cultured in DMEM/Ham's F-12 (1:1), supplemented with $10 \%$ FBS, insulin $(0.005 \mathrm{mg} /$ $\mathrm{ml})$, transferrin $(0.01 \mathrm{mg} / \mathrm{ml})$, sodium selenite $(30 \mathrm{nM})$, hydrocortisone (10 nM), beta-estradiol (10 nM), HEPES $(10 \mathrm{mM})$, and L-glutamine $(2 \mathrm{mM})$. Both cell lines were cultured at $37{ }^{\circ} \mathrm{C}$ in $5 \% \mathrm{CO}_{2}$.

\section{Immunofluorescence}

Transfected NCI-H82 cells (on chamber slides) were fixed in $4 \%$ paraformaldehyde. Primary and secondary antibodies (IA-2; 1:100 dilution), and Alexa Fluor 568 Goat Anti-Rabbit IgG (H+L) (1:500 dilution), respectively, were used. VECTASHIELD mounting medium with DAPI (Vector Laboratories) was used for nuclear staining. Images were captured with ZEN Imaging Software (ZEISS) using constant exposure parameters for each fluorescence channel. Images shown are representative field of three independent experiments.

\section{IA-2 siRNA transfection}

A total of $2 \times 10^{4}$ cells/well were seeded in 24-well culture plates 1 day prior to undergoing transfection with either the IA-2 siRNAs (Hs_PTPRN_3, 4, 5, 6), or the scramble siRNA with the HiPerFect Transfection Reagent for $10 \mathrm{~min}$ (according the manufacturer's instruction). Briefly, cells were resuspended in $100 \mu \mathrm{l}$ of fresh culture medium (containing serum and antibiotics), and then $100 \mu \mathrm{l}$ of the siRNA transfection complexes solution (100 $\mathrm{nM}$ at final concentration after adding $400 \mu \mathrm{l}$ culture medium) was added drop-wise onto the cells. The plates were then gently swirled to ensure uniform distribution of the transfection complexes. The suspension was incubated at normal growth conditions for $6 \mathrm{~h}$, and then $400 \mu \mathrm{l}$ of the culture medium (containing serum and antibiotics) was added to the cells and incubated until further analysis.

\section{MicroRNA mimic and inhibitor transfection}

Cells were transfected with the miR-342 mimic or inhibitor, or with the scramble negative control using the HiPerFect Transfection Reagent (according to the manufacturer's instructions), and incubated for 10 min further analysis. First, cells $\left(2 \times 10^{4}\right.$ cells/well $)$ were transferred into 24-well plates after overnight culture. The miRNA mimic, miRNA inhibitor, or the control was diluted in $100 \mu \mathrm{l}$ culture medium without serum (a final miRNA mimic concentration of $5 \mathrm{nM}$, or a final miRNA inhibitor concentration of $50 \mathrm{nM}$ ). Subsequently, the HiPerFect Transfection Reagent $(3 \mu \mathrm{l})$ was added into the diluted miRNA mimic/inhibitor solution, and then vortexed. After $8 \mathrm{~min}$, the solution was incubated for $10 \mathrm{~min}$ at room temperature, and then the complexes were added 
onto the cells. Finally, the cells were incubated with the transfection complexes under their normal growth conditions until further analysis.

\section{Cell growth assay}

Cell proliferation was tested using the Cell Counting Kit-8 (CCK-8) assay according to the manufacturer's instructions (Sigma-Aldrich, St. Louis, MO). In brief, after the cells were subjected to the different treatments (transfection with IA-2 siRNA or miR-342 mimic or inhibitor), they were cultured for up to 4 days. Cells were collected each day and then transferred into 96-well plates. Subsequently, CCK-8 $(10 \mu \mathrm{l})$ was added to each well, and then the cells were cultured for another $3 \mathrm{~h}$. Cell density was determined by measuring the absorbance at $450 \mathrm{~nm}$ using a VersaMax Microplate Reader (Molecular Devices, USA).

\section{ACh secretion and cell content assay}

After transfecting the NCI-H82 and NCI-H345 cells with either the IA-2 siRNA, miR-342 mimic, or the miR-342 inhibitor, the cells were incubated for 4 days, and then the cells and supernatants were collected [6,7]. ACh level was measured using a human acetylcholine ELISA kit according to manufacturer's protocol. For determination of ACh release, cell suspensions were collected with $5 \times 10^{-5} \mathrm{M}$ neostigmine. For measurement of ACh contents, cells were washed in $1 \times$ PBS, counted, and then sonicated in ice-cold phosphate buffer ( $\mathrm{pH}$ 7.4) containing $10^{-2} \mathrm{M}$ of the acetylcholinesterase inhibitor neostigmine. All samples were centrifuged at $1000 \mathrm{rpm}$ for $2 \mathrm{~min}$ at $4{ }^{\circ} \mathrm{C}$; supernatants were then immediately assayed for determination of $\mathrm{ACh}$ levels.

\section{Total RNA, miRNA, and quantitative PCR}

Total RNA and miRNA were extracted using the miRNeasy Mini kit following the manufacturer's protocol. Quantitative RT-PCR for both mRNA and miRNA was performed using the miScript II RT kit, and then the miScript SYBR Green PCR kit for measuring miR342 expression, or SYBR Green PCR Master Mix for measuring the expression of IA-2 and GAPDH (glyceraldehyde-3-phosphate dehydrogenase) mRNA with a 7500 Real-Time PCR system (Applied Biosystems). IA-2 primer sequences used in PCR analysis, forward: 5'-ACGCTCACGCAGTTCCACTT-3', reverse: 5'-GCGATGTCAATCTCCTTCAC-3'; GAPDH primer sequences, forward: 5'-GAGAACGGGAAGCTTGT

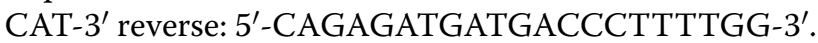

\section{Protein extraction and western blot analysis}

Proteins from cells were isolated by M-PER Mammalian Protein Extraction Reagent. Protein concentrations were quantified; samples were mixed with reducing loading buffer, and then heated at $70{ }^{\circ} \mathrm{C}$ for $10 \mathrm{~min}$. Equal amounts of protein was loaded into each lane, and separated by electrophoresis. Proteins were subsequently transferred onto a PVDF (polyvinylidene fluoride) membrane and then blocked in blocking buffer (PBS/0.1\% Tween20 (PBS-T)/5 \% nonfat-milk). After blocking for $2 \mathrm{~h}$, blots were incubated over night at $4{ }^{\circ} \mathrm{C}$ with the antiIA-2 N-terminal antibody (\#49) or $\alpha$-tubulin (1:5000 dilution). After washing, the blots were incubated for 30 min at room temperature with HRP (horseradish peroxidase) conjugated secondary antibodies. The blots were then washed and films were subsequently developed. Blots were quantitated using the NIH Image J Software.

\section{ACh rescues $\mathrm{NCl}-\mathrm{H} 82$ cell growth}

Different concentrations of ACh $(0,1,10,100,300,1$, 3 , and $10 \mathrm{mM}$ ) were added to the NCI-H82 cells, and 4 days later, a cell growth assay was performed. The optimal concentration of ACh was selected, and then added to the NCI-H82 cells treated with scramble siRNA, IA-2 siRNA, miR-342 mimic, or the miR-342 inhibitor. Four days later, a cell growth assay was performed to evaluate the rescue effect of each treatment condition.

\section{Statistical analysis}

All experiments were performed in triplicate and the data represent the mean \pm SEM. The Student's $t$ test and Oneway ANOVA were used to determine statistical significance. In all cases, a $\mathrm{P}<0.05$ was considered significant.

\section{Results}

Downregulation of IA-2 by siRNA suppresses SCLC cells growth as well as ACh cell content and secretion

NCI-H82 cells were treated with IA-2 siRNA (Hs PTPRN_3, 4, 5, 6) and then IA-2 mRNA and IA-2 protein levels were measured by quantitative real-time PCR (qRT-PCR) and Western Blot. Both IA-2 mRNA and protein were substantially decreased (Fig. 1a-c). Immunostaining with the IA-2 antibody confirmed the reduction of IA-2 expression after transfecting the cells with the IA-2 siRNA (Fig. 1d).

To elucidate the role of IA-2 on SCLC growth, we knocked down endogenous IA-2 in NCI-H82 and NCIH345 cells with IA-2 siRNA. A significant reduction in cell proliferation was observed in both cell lines, with the greatest reduction occurring in the NCI-H82 cell line (60\%), compared to the NCI-H345 cell line (40\%) at 4 days post-transfection (Fig. 2a, b). Previous reports showed that ACh acts as an autocrine growth factor. To investigate whether IA-2 could regulate ACh content and secretion in SCLC cells, both were measured by ELISA after cells were transfected with IA-2 siRNA for 4 days. 
(a)

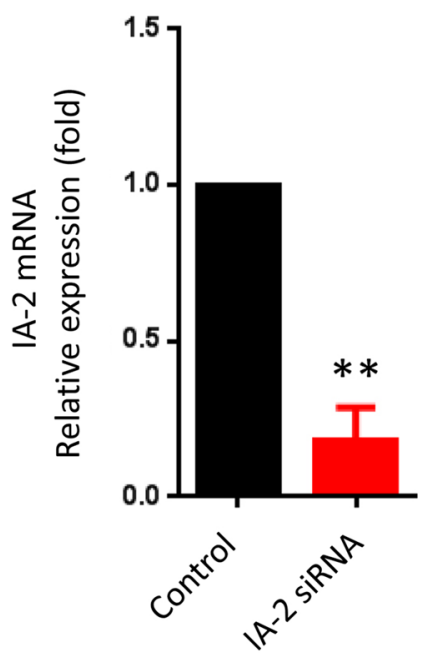

(b)

$$
\mathrm{NCl}-\mathrm{H} 82
$$

IA-2

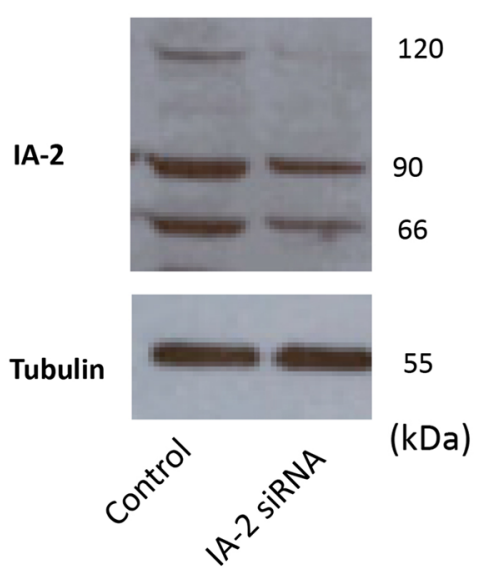

(c)

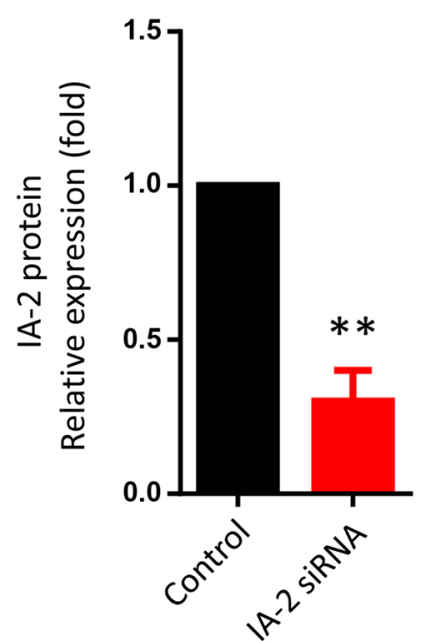

(d)

IA-2

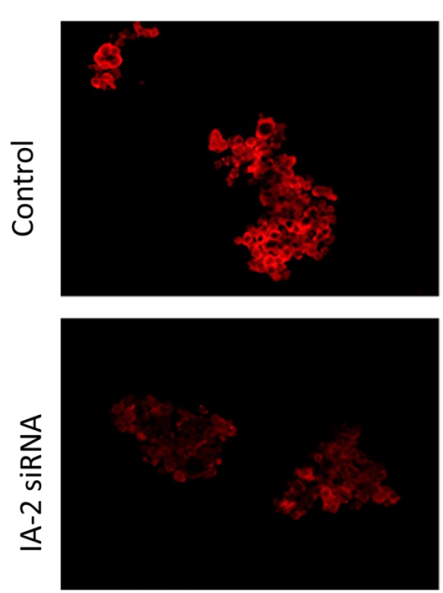

Fig. 1 IA-2 expression is decreased by siRNA. a The knockdown of endogenous IA-2 mRNA by siRNA (determined by quantitative RT-PCR analysis), was reduced $\sim 80 \%$ in $\mathrm{NCI}$-H82. b Western blot shows that protein levels for IA-2 (as evaluated by anti-IA-2 N-terminal \#49 antibody), was also significantly reduced. Three major bands of IA-2 are detected as indicated due to post-translational modifications [33]. c IA-2 protein level was reduced $\sim 70 \%$ as determined by the intensity of the $90-\mathrm{kDa}$ bands using the NIH Image J program. $\mathbf{d}$ Immunostaining with the IA-2 N-terminal antibody shows that the level of IA-2 in IA-2-specific RNAi treated NCl-H82 cells was significantly lower than the control cells. Data (mean \pm SE) were derived from three independent experiments (in triplicate), and normalized to GAPDH mRNA levels. ${ }^{* * P}<0.01$

As seen in Fig. 2c and e, both ACh cell content and ACh secretion were decreased by $\sim 20$ and $\sim 30 \%$, respectively in NCI-H82 cells, as compared to the scramble siRNA control. Similar results were observed with NCI-H345 cells (Fig. 2d, f).

\section{miR-342 regulates IA-2 expression in SCLC cells}

To see if the expression of IA-2 is regulated by miRNAs, NCI-H82 and NCI-H345 cells were transfected with a miR-342 mimic or miR-342 inhibitor. As seen in Fig. 3, the miR-342 mimic significantly decreased the expression of IA-2 mRNA, whereas the miR-342 inhibitor significantly enhanced the expression of IA-2 mRNA in both cell lines (Fig. 3a, b). Western blot analysis showed that miR-342 mimic decreased, whereas miR-342 inhibitor enhanced protein expression in both cell lines (Fig. 3c-f).

\section{MiR-342 mimic suppresses IA-2 expression, SCLC cell growth and $\mathrm{ACh}$ content and secretion}

Given that miR-342 directly targets IA-2 as previously demonstrated in the insulin-containing MIN6 cells by "RNA immunoprecipitation PCR with anti-argonaute 2" [25], the impact of miR-342 on SCLC cell proliferation 


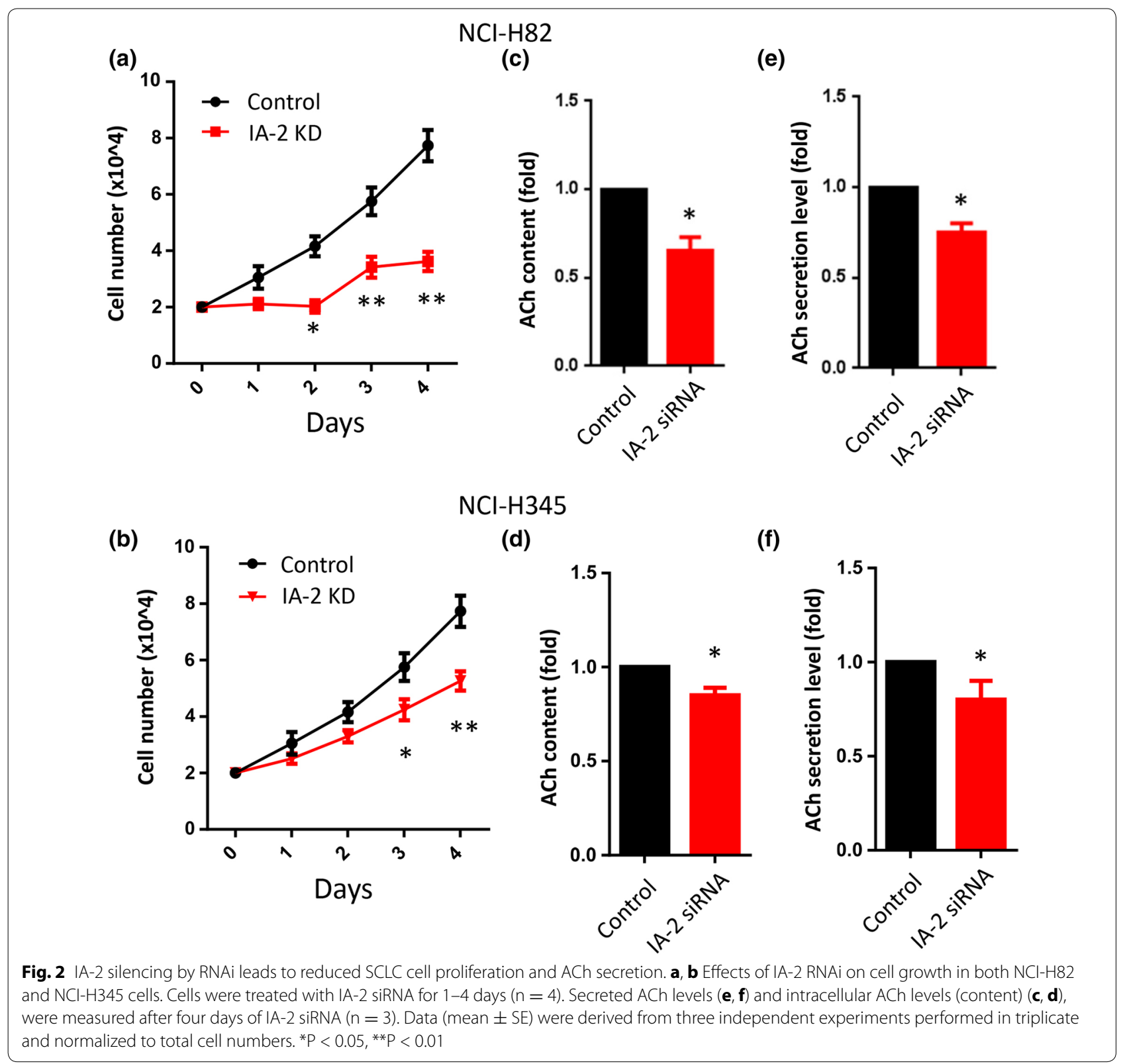

was evaluated. Growth curve analysis showed that NCIH82 and NCI-H345 cells treated with the miR-342 mimic significantly suppressed SCLC cell proliferation by as much as $30 \%$ (Fig. 4a, b). In contrast, the miR-342 inhibitor produced a slight but significant increase in SCLC cell proliferation at 4 days after transfection (Fig. 4a, b).

To determine the effect of miR-342 on ACh cell content and secretion, SCLC cells were transfected with a miR-342 mimic or inhibitor. Both NCI-H82 and NCIH345 cells transfected with the miR-342 mimic showed a significant reduction in ACh cell content and secretion (Fig. 4c-f). NCI-H82 cells treated with the miR-342 inhibitor showed a slight but not significant increase in ACh secretion (Fig. 4e-f), and a barely significant increase in ACh content (Fig. 4c).

ACh rescues the inhibitory effects IA-2 siRNA or miR-342 mimic on SCLC proliferation

To confirm that the effect of IA-2 siRNA and miR-342 on SCLC cell proliferation is mediated through the ACh autocrine growth loop, $\mathrm{ACh}$ was added to the culture medium of the NCI-H82 cells that were treated with either the scrambled siRNA, IA-2 siRNA, miR-342 mimic, or the miR-342 inhibitor. As seen in Fig. 5a, the addition of ACh to control cells resulted in significantly increasing the amount of cell proliferation by as much as $50 \%$. 


\section{$\mathrm{NCl}-\mathrm{H} 82$}

(a)

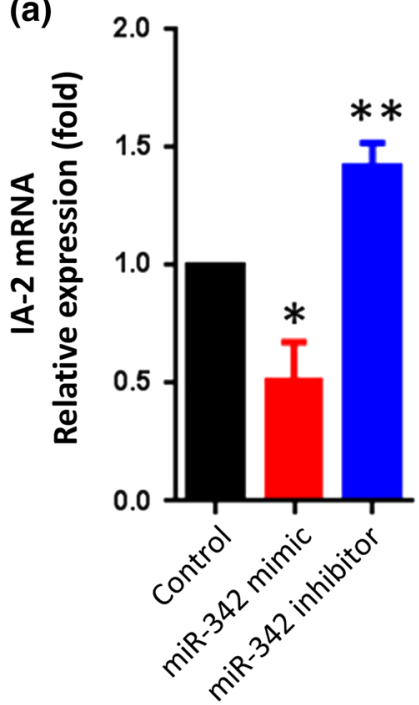

(c)

IA-2

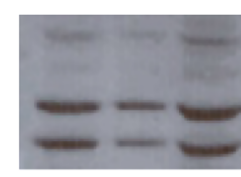

$\boldsymbol{\alpha}$-tubulin

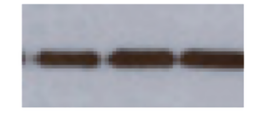

120

90

66

55

(kDa)

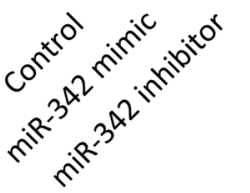

(e)

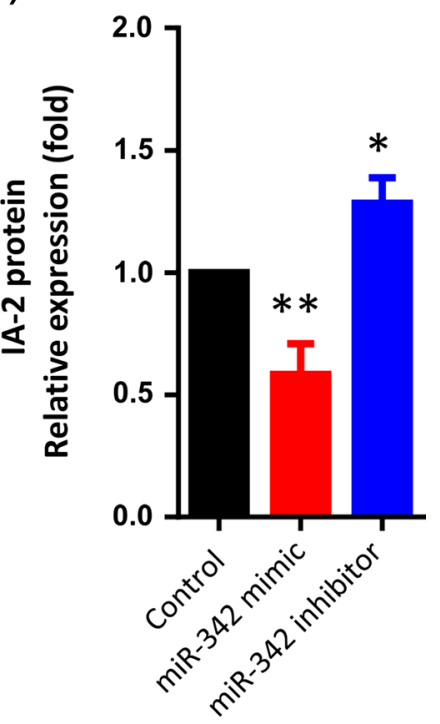

\section{$\mathrm{NCl}-\mathrm{H} 345$}

(d)

(b)

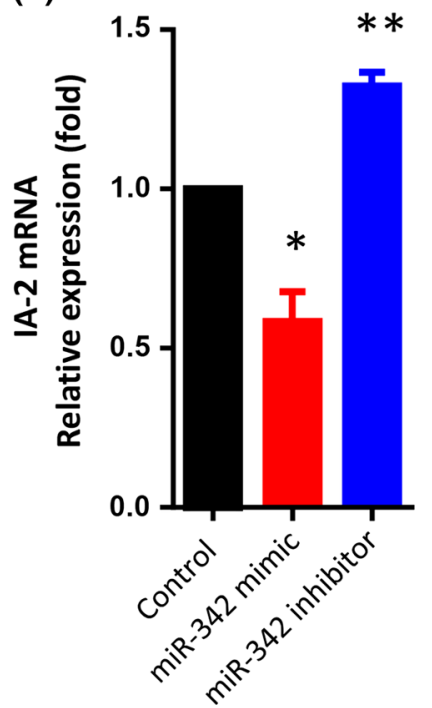

(f)

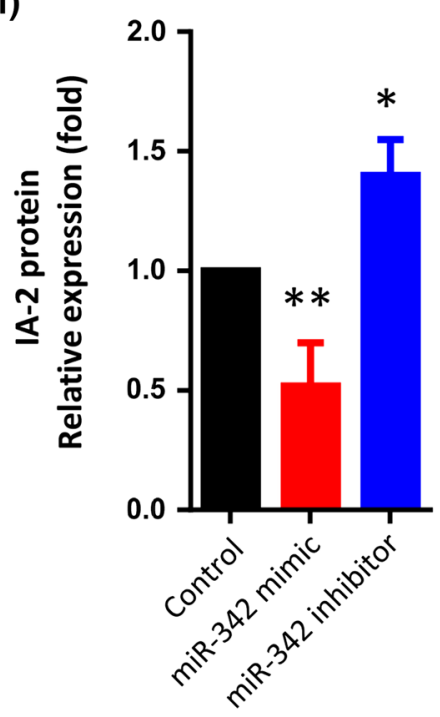

Fig. 3 miR-342 regulates IA-2 expression level in SCLC cells. a, b Effects of miR-342 mimic and miR-342 inhibitor on IA-2 expression in NCI-H82 and $\mathrm{NCl}-\mathrm{H} 345$ cells $(n=4)$, as quantitated by Real-Time PCR. Relative mRNA levels were normalized to GAPDH. c, d Protein levels for IA-2 in NCl-H82 and NCl-H345 cells $(n=4)$ were quantitated by Western blotting using the anti-IA-2 antibody. e, f Relative expression levels of IA-2 in Western blots (normalized by a-tubulin), were determined by $\mathrm{NIH}$ Image J. ${ }^{*} \mathrm{p}<0.05 ;{ }^{* *} \mathrm{p}<0.01$

An even greater increase in proliferation was observed when IA-2 siRNA treated cells were incubated with ACh (1 mM, optimal concentration). Similarly, ACh produced a highly significant increase in the proliferation of cells that had been treated with miR-342 mimic (Fig. 5b). Finally, the addition of ACh to cells that had been treated with the miR-342 inhibitor produced an increase in cell proliferation with the greatest magnitude compared to the other treatment conditions (Fig. 5b). Taken together, these findings show for the first time, that the neurotransmitter ACh is effective in rescuing the inhibitory effects of siRNA and miR-342 on cell proliferation. 


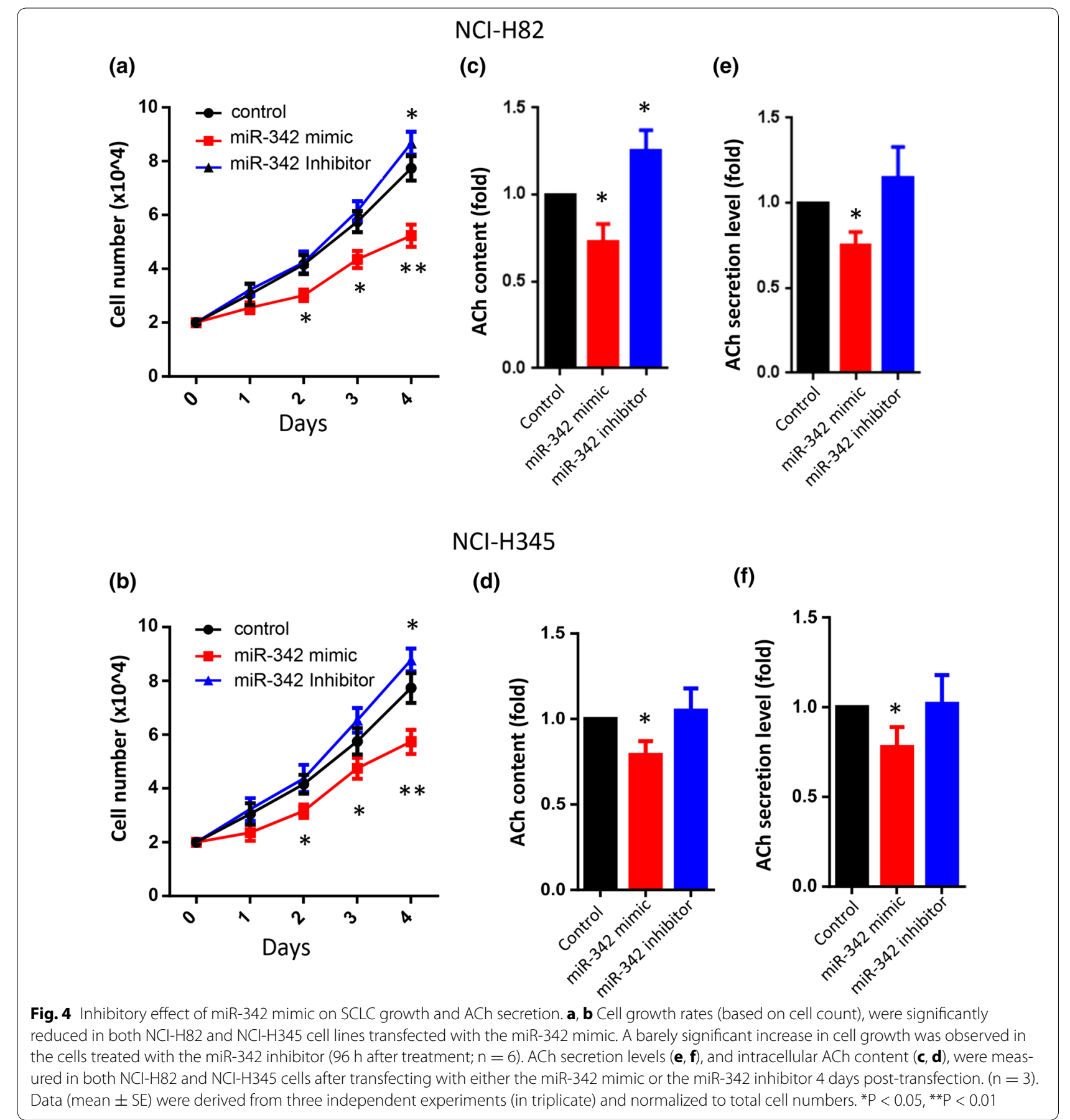

\section{Discussion}

Our previous studies demonstrated that IA-2 is highly expressed in multiple human SCLC cell samples [18], but not in normal lung tissue. Although IA-2 has been well characterized in many neuroendocrine cells, its characterization and function in SCLC have so far not been determined. In this study, we found that high expression of IA-2 is associated with the growth and proliferation of SCLC cells. Conversely, we also demonstrated that the knockdown of IA-2 expression by RNAi can significantly reduce the growth rate of SCLC cells.

SCLC cells have the capacity to synthesize and secrete ACh $[6,7]$ and a cholinergic autocrine loop positively regulates SCLC cell growth $[6,26]$. On the cytoplasmic 

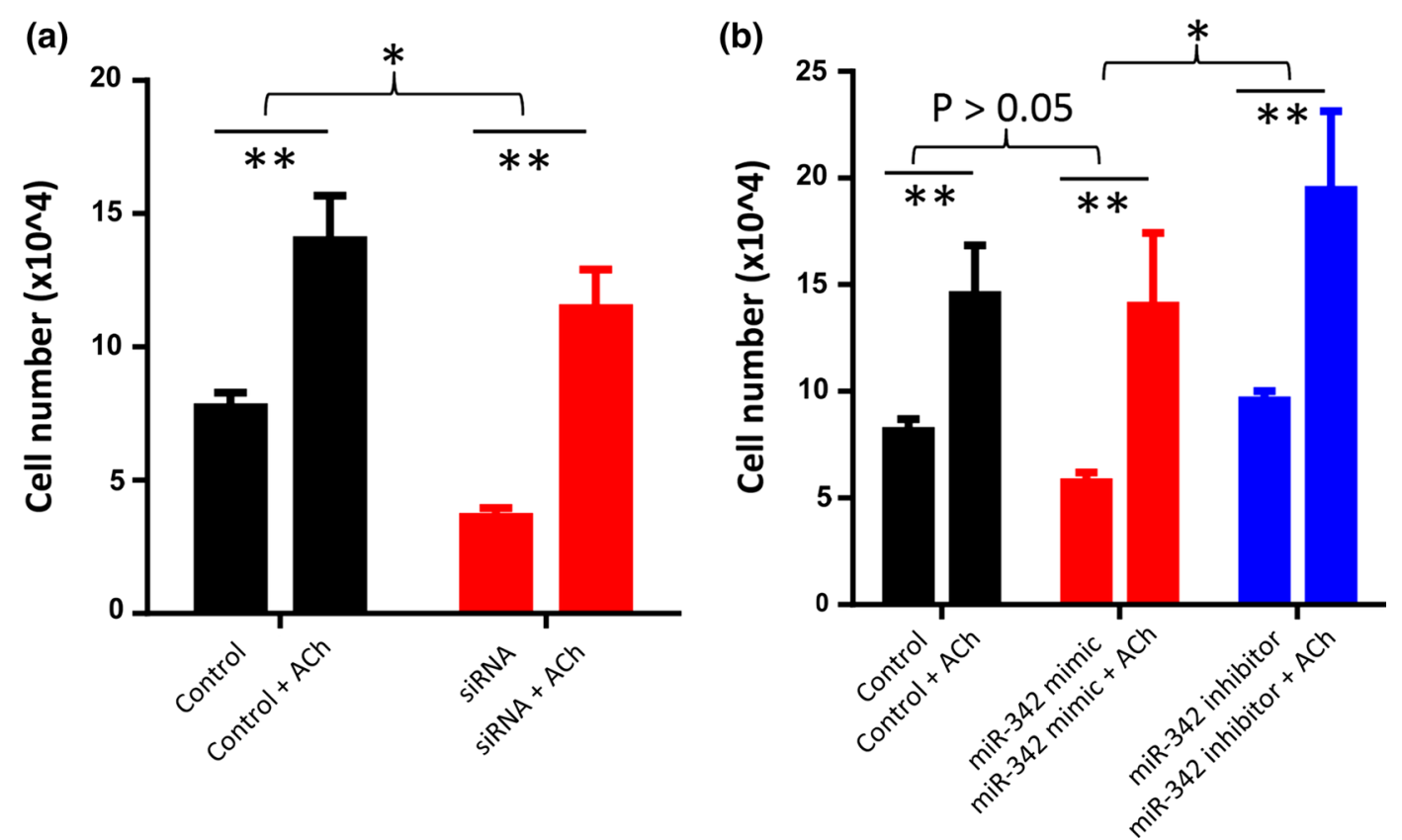

Fig. 5 ACh rescues the inhibitory effect of IA-2 siRNA and miR-342 mimic on SCLC cell growth. a Treatment of SCLC cells with IA-2 siRNA inhibits cell growth. In contrast, treatment of $\mathrm{NCl}-\mathrm{H} 82$ cells with $\mathrm{ACh}(1 \mathrm{mM})$, rescues cell growth. The increase of proliferation in the IA-2 siRNA and ACh treated cells is significantly higher than that incubated with ACh. b Similarly, the inhibitory effect of miR-342 on NCl-H82 cell growth is reduced by ACh treatment ( $1 \mathrm{mM}$ ). Similarly, the addition of ACh to cells treated with the miR-342 inhibitor produced an increase in cell proliferation with the greatest magnitude compared to the other treatment conditions. $n=4 ;{ }^{*} P<0.05$, ${ }^{* *} P<0.01$

membrane, two types of $\mathrm{ACH}$ receptors have been found: nAChR ligand-gated ion channels $(\alpha 3, \alpha 5, \alpha 7, \beta 2$ and $\beta 4)$ and $G$ protein-coupled receptors $\mathrm{mAChR}$ (M3 and M5) [6, 27-30]. Moreover, the knockdown of choline transporter-like protein 4 (CTL4), which mediates ACh synthesis and secretion, has been shown to significantly depress the growth of SCLC (NCI-H82) cells without affect choline uptake [31].
In the present study, we showed that silencing of IA-2 results in a substantial decrease in SCLC cell growth secondary to a decrease in the content and secretion of ACh (Fig. 6). The silencing of IA-2 has previously been shown to decrease the stability and number of DCV and, in turn, the secretion of hormones and neurotransmitters. In the present study, we also showed that overexpression of the miR-342, which targets the $3^{\prime}$-UTR (untranslated

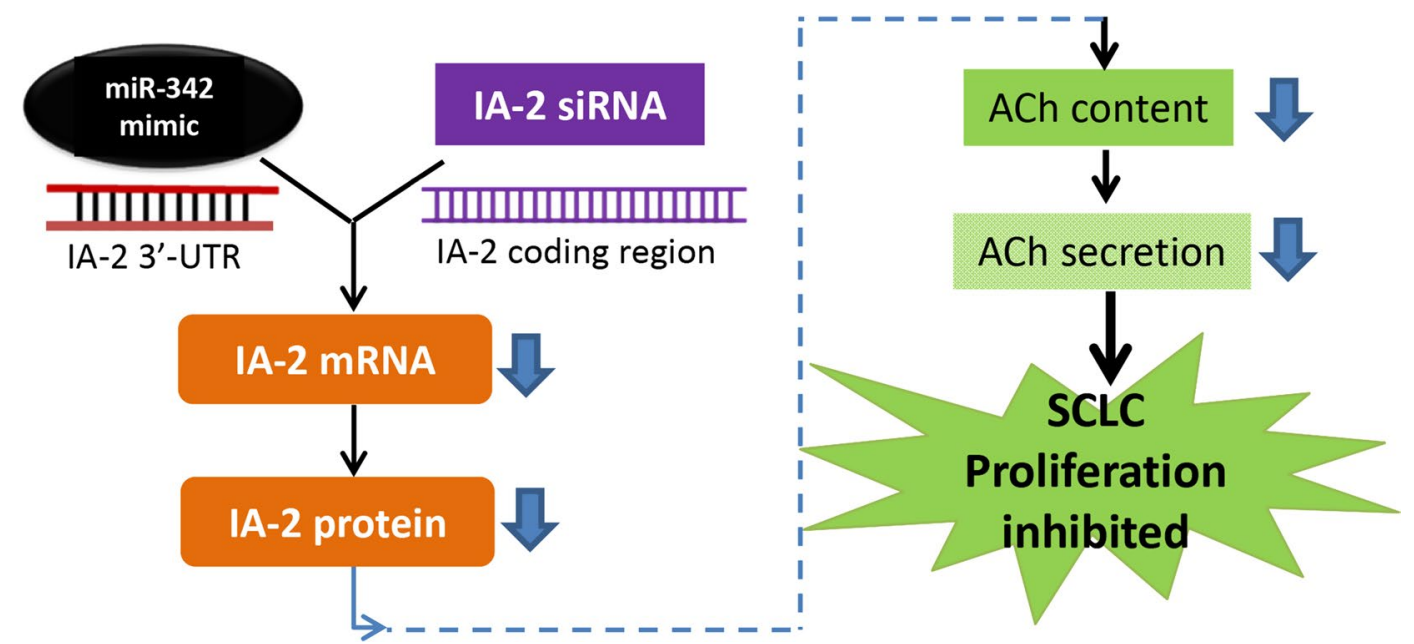

Fig. 6 Schematic representation of the effects of IA-2 siRNA and miR-342 mimic on SCLC cell proliferation through ACh autocrine function 
region) of IA-2, results in a decrease in the expression of IA-2 mRNA and, in turn, a decrease in the cellular content and secretion of ACh resulting in a decrease in the growth of SCLC cells (Fig. 6). Further support for the idea that the decrease in $\mathrm{ACh}$ is responsible for the decrease in SCLC cell growth was obtained by restoring cell growth to nearly normal levels by treatment with ACh.

SCLC is an aggressive malignancy with a distinct natural history and poor prognosis, leading to the death of approximately 16,000 patients per year in United States. For decades, cytotoxic chemotherapy has remained the backbone of treatment since SCLC is a chemo-sensitive tumor. Nonetheless, high response rates are not usually translated into cure. In spite of recent advances in elucidating aberrant molecular pathways and new therapeutic targets, no significant improvement in patients' survival has been demonstrated [32].

\section{Conclusion}

Taken together, the demonstration that both miR-342 and its target gene IA-2 are involved in the growth of SCLC cells, through their effect on the secretion of acetylcholine, represents a novel approach that may be therapeutically useful in the treatment of SCLC tumors.

\begin{abstract}
Abbreviations
ACh: acetylcholine; ACTH: adrenocorticotropic hormone; CCK-8: cell counting kit-8; DAPI: 4',6-diamidino-2-phenylindole, dihydrochloride; DCV: dene-core vesicle; ELISA: enzyme-linked immunosorbent assay; FSH: follicular stimulating hormone; GAPDH: glyceraldehyde-3-phosphate dehydrogenase; NIH: National Institutes of Health; HRP: horseradish peroxidase; IA-2: islet cell antigen-2; ICA512: islet cell antigen 512; LH: luteinizing hormone; NIDCR: National Institute of Dental and Craniofacial Research; NE: norepinephrine; NSCLC non-small cell lung cancer; Phogrin: phosphatase homologue in granules of insulinoma; POMC: pro-opiomelanocortin; PTPRN: protein tyrosine phosphatase, receptor type N; PVDF: polyvinylidene fluoride; SCLC: small cell lung cancers; UTR: untranslated region.
\end{abstract}

\section{Authors' contributions}

HX and TC participated in the experiment design, data acquisition and analysis as well as drafting the manuscript. GNC and LA analyzed data and revised the manuscript. ALN conceived of the study and revised the manuscript. All authors read and approved the final manuscript.

\section{Competing interests}

The authors declare that that they have no competing interests.

\section{Availability of data and materials}

This manuscript does not have new software or application/tool to share. Raw data are available for sharing if needed.

\section{Funding}

This work was supported by the Intramural Research Program of the NIDCR, $\mathrm{NIH}$. This work was supported by the Intramural Research Program of the $\mathrm{NIDCR}, \mathrm{NIH}$. No additional external funding was received for this study. The funder had no role in the study design, data collection and analysis, decision to publish, or preparation of the manuscript.

Received: 25 July 2016 Accepted: 16 September 2016

Published online: 26 September 2016
References

1. Ettinger DS, Wood DE, Akerley W, Bazhenova LA, Borghaei H, Camidge DR, Cheney RT, Chirieac LR, D'Amico TA, Demmy TL, et al. Non-small cell lung cancer, version 1.2015. J Natl Compr Cancer Netw. 2014;12:1738-61.

2. Kalemkerian GP, Akerley W, Bogner P, Borghaei H, Chow LQ, Downey RJ, Gandhi L, Ganti AK, Govindan R, Grecula JC, et al. Small cell lung cancer. J Natl Compr Cancer Netw. 2013;11:78-98.

3. Goldstraw P, Ball D, Jett JR, Le Chevalier T, Lim E, Nicholson AG, Shepherd FA. Non-small-cell lung cancer. Lancet. 2011;378:1727-40.

4. van Meerbeeck JP, Fennell DA, De Ruysscher DK. Small-cell lung cancer. Lancet. 2011;378:1741-55.

5. Teicher BA. Targets in small cell lung cancer. Biochem Pharmacol. 2014;87:211-9.

6. Song PF, Sekhon HS, Jia YB, Keller JA, Blusztajn JK, Mark GP, Spindel ER. Acetylcholine is synthesized by and acts as an autocrine growth factor for small cell lung carcinoma. Cancer Res. 2003;63:214-21.

7. Song P, Sekhon HS, Proskocil B, Blusztajn JK, Mark GP, Spindel ER. Synthesis of acetylcholine by lung cancer. Life Sci. 2003;72:2159-68.

8. Stewart MF, Crosby SR, Gibson S, Twentyman PR, White A. Small cell lung cancer cell lines secrete predominantly ACTH precursor peptides not ACTH. Br J Cancer. 1989;60:20-4.

9. Stovold R, Blackhall F, Meredith S, Hou J, Dive C, White A. Biomarkers for small cell lung cancer: neuroendocrine, epithelial and circulating tumour cells. Lung Cancer. 2012;76:263-8.

10. Nishimura T, Kubosaki A, Ito Y, Notkins AL. Disturbances in the secretion of neurotransmitters in IA-2/IA-2beta null mice: changes in behavior, learning and lifespan. Neuroscience. 2009;159:427-37.

11. Solimena M, DirkX R Jr, Hermel JM, Pleasic-Williams S, Shapiro JA, Caron L, Rabin DU. ICA 512, an autoantigen of type I diabetes, is an intrinsic membrane protein of neurosecretory granules. EMBO J. 1996;15:2102-14.

12. Nishimura T, Harashima S, Yafang H, Notkins AL. IA-2 modulates dopamine secretion in PC12 cells. Mol Cell Endocrinol. 2010;315:81-6.

13. Kim SM, Theilig F, Qin Y, Cai T, Mizel D, Faulhaber-Walter R, Hirai H, Bachmann S, Briggs JP, Notkins AL, Schnermann J. Dense-core vesicle proteins IA-2 and IA-2\{beta\} affect renin synthesis and secretion through the \{beta\}-adrenergic pathway. Am J Physiol Ren Physiol. 2009;296:F382-9.

14. Saeki K, Zhu M, Kubosaki A, Xie J, Lan MS, Notkins AL. Targeted disruption of the protein tyrosine phosphatase-like molecule IA-2 results in alterations in glucose tolerance tests and insulin secretion. Diabetes. 2002;51:1842-50.

15. Kubosaki A, Nakamura S, Notkins AL. Dense core vesicle proteins IA-2 and IA-2beta: metabolic alterations in double knockout mice. Diabetes. 2005;54(Suppl 2):S46-51.

16. Kubosaki A, Nakamura S, Clark A, Morris JF, Notkins AL. Disruption of the transmembrane dense core vesicle proteins IA-2 and IA-2beta causes female infertility. Endocrinology. 2006;147:811-5.

17. Cai T, Hirai H, Zhang G, Zhang M, Takahashi N, Kasai H, Satin LS, Leapman RD, Notkins AL. Deletion of la-2 and/or la-2beta in mice decreases insulin secretion by reducing the number of dense core vesicles. Diabetologia. 2011;54:2347-57.

18. Xie H, Notkins AL, Lan MS. IA-2, a transmembrane protein tyrosine phosphatase, is expressed in human lung cancer cell lines with neuroendocrine phenotype. Cancer Res. 1996;56:2742-4.

19. Lan MS, Lu J, Goto Y, Notkins AL. Molecular cloning and identification of a receptor-type protein tyrosine phosphatase, IA-2, from human insulinoma. DNA Cell Biol. 1994;13:505-14.

20. Torii S, Saito N, Kawano A, Hou N, Ueki K, Kulkarni RN, Takeuchi T. Gene silencing of phogrin unveils its essential role in glucose-responsive pancreatic beta-cell growth. Diabetes. 2009;58:682-92.

21. Mziaut H, Kersting S, Knoch KP, Fan WH, Trajkovski M, Erdmann K, Bergert H, Ehehalt F, Saeger HD, Solimena M. ICA512 signaling enhances pancreatic beta-cell proliferation by regulating cyclins D through STATs. Proc Natl Acad Sci USA. 2008;105:674-9.

22. Kertesz M, lovino N, Unnerstall U, Gaul U, Segal E. The role of site accessibility in microRNA target recognition. Nat Genet. 2007;39:1278-84.

23. Dacic S, Kelly L, Shuai Y, Nikiforova MN. miRNA expression profiling of lung adenocarcinomas: correlation with mutational status. Mod Pathol. 2010;23:1577-82.

24. Angelloz-Nicoud P, Binoux M. Autocrine regulation of cell proliferation by the insulin-like growth factor (IGF) and IGF binding protein-3 protease system in a human prostate carcinoma cell line (PC-3). Endocrinology. 1995;136:5485-92. 
25. Abuhatzira L, Xu H, Tahhan G, Boulougoura A, Schaffer AA, Notkins AL. Multiple microRNAs within the 14q32 cluster target the mRNAs of major type 1 diabetes autoantigens IA-2, IA-2beta, and GAD65. FASEB J. 2015;29:4374-83.

26. Song P, Spindel ER. Basic and clinical aspects of non-neuronal acetylcholine: expression of non-neuronal acetylcholine in lung cancer provides a new target for cancer therapy. J Pharmacol Sci. 2008;106:180-5.

27. Song P, Sekhon HS, Fu XW, Maier M, Jia Y, Duan J, Proskosil BJ, Gravett C, Lindstrom J, Mark GP, et al. Activated cholinergic signaling provides a target in squamous cell lung carcinoma. Cancer Res. 2008;68:4693-700.

28. Song P, Sekhon HS, Lu A, Arredondo J, Sauer D, Gravett C, Mark GP, Grando SA, Spindel ER. M3 muscarinic receptor antagonists inhibit small cell lung carcinoma growth and mitogen-activated protein kinase phosphorylation induced by acetylcholine secretion. Cancer Res. 2007:67:3936-44
29. Resende RR, Adhikari A. Cholinergic receptor pathways involved in apoptosis, cell proliferation and neuronal differentiation. Cell Commun Signal. 2009;7:20.

30. Ami N, Koga K, Fushiki H, Ueno Y, Ogino Y, Ohta H. Selective M3 muscarinic receptor antagonist inhibits small-cell lung carcinoma growth in a mouse orthotopic xenograft model. J Pharmacol Sci. 2011;116:81-8.

31. Song P, Rekow SS, Singleton CA, Sekhon HS, Dissen GA, Zhou M, Campling B, Lindstrom J, Spindel ER. Choline transporter-like protein 4 (CTL4) links to non-neuronal acetylcholine synthesis. J Neurochem. 2013;126:451-61.

32. Koinis F, Kotsakis A, Georgoulias V. Small cell lung cancer (SCLC): no treatment advances in recent years. Transl Lung Cancer Res. 2016;5:39-50.

33. Xie H, Deng YJ, Notkins AL, Lan MS. Expression, characterization, processing and immunogenicity of an insulin-dependent diabetes mellitus autoantigen, IA-2, in Sf-9 cells. Clin Exp Immunol. 1998;113:367-72.

\section{Submit your next manuscript to BioMed Central and we will help you at every step:}

- We accept pre-submission inquiries

- Our selector tool helps you to find the most relevant journal

- We provide round the clock customer support

- Convenient online submission

- Thorough peer review

- Inclusion in PubMed and all major indexing services

- Maximum visibility for your research

Submit your manuscript at www.biomedcentral com/submit 